\title{
Energy distribution in disordered elastic networks
}

\author{
Gustavo R. Plaza \\ Departamento de Ciencia de Materiales, ETSI de Caminos, Canales y Puertos, Universidad Politécnica de Madrid, 28040 Madrid, Spain
}

(Received 8 June 2010; revised manuscript received 2 August 2010; published 8 Seplember 2010)

\begin{abstract}
Disordered networks are found in many natural and artificial materials, from gels or cytoskeletal structures to metallic foams or bones. Here, the energy distribution in this type of networks is modeled, taking into account the orientation of the struts. A correlation between the onientation and the energy per unit volume is found and described as a function of the connectivity in the network and the relative bending stiffness of the struts. If one or both parameters have relatively large values, the struts aligned in the loading direction present the highest values of energy. On the contrary, if these have relatively sinall values, the highest values of energy can be reached in the struts oriented transversally. This result allows explaining in a simple way remodeling processes in biological materials, for example. the remodeling of trabecular bone and the reorganization in the cytoskeleton. Additionally. the correlation between the orientation, the affinity. and the bending-stretching ratio in the network is discussed.
\end{abstract}

\section{INTRODUCTION}

Spongy cellular and fibrillar materials are the basis of a wide variety of systems which require bearing loads and/or fulfilling spaces at low cost of mass while allowing the storage and How of snbstances inside. These materials consist of a network of interconnected strnts or slabs and can be fonnd in natnre as well as synthesized artificially for specific applications. The properties of these networks are determined by the characteristics of the struts or slabs and the type of connectivity between thein. In the case of certain biological materials these properties will in turn yield to a directed remodeling of their architecture as a consequence of the mechanical stresses that they are subjected to. Annong the wide variety of materials that can be mentioned (aluminum foams, collagenous tissues, wood, honeycombs, etc.) two biological ones are of particnlar interest, namely, the cytoskeleton of cells and the cancellons bone.

The cytoskeleton is a network in the cytoplasm that determines the mechanical behavior of cells. It plays an important role in mechanotransduction or motility of cells. The cytoskeleton is composed of macromolecules (actin filaments, interinediate filaments, and nicrotubules) forming a disordered network of semiflexible polymers [1] due to bolh the bending and longitudinal stiffness of the filannents. Many authors have investigated the properties of the cytoskeleton by means of micromechanical models [2-11]. These are expected to be usefnl for the understanding, for instance, of mechanotransduction, tissue development, or anomalons behavior of ill cells in comparison with healthy ones [12-14]. As part of the cytoskeleton, the actin filaments form a dynanic network [15] with assembling and disassembling rates that depend on the mechanical stimuli. It has been shown that by applying a cyclic stretching the filaments align perpendicularly to the direction of stretching forming bundles called stress fibers [16]. Further, the stability of these bundles over the time has been inodeled. However, the mechanisin that explains the evolution from the initial disordered network to the final bundle state has not been identified so far.

In the case of cancellous bone, also known as trabecular bone, the network is composed of calcified connective tissue in the form of struts or slabs naned trabeculae. The vertebrae of the spine and the end parts of long bones are constituted of this kind of porons bone. It has been observed that the predominant trabeculae orientation corresponds with that of the maximnm compressive and/or tensile stress directions in a compact material with the same shape and subjected to equivalent forces. This phenomenon is known as Wolf's law $[17,18]$. This trabeculae orientation is the result of an adaptation to the loading conditions. According to the so-called remodeling mechanism $[19,20]$, the bone mass increases at those places of high enough strain energy and, on the contrary, it decreases in the places of low one. This mechanism allows for adaptation to changes in mechanical loading. The relation between the remodeling mechanisin and the trabeculae orientation has been simulated so far by complex iterative numerical models [20] that involve parameters such as elastic moduli, bone formation and resorption rates, etc. Nevertheless, these models based on iterative processes do not express the essence of the fundamental mechanical origin of Wolf's law in a simpler pictnre.

Back to the general disordered elastic networks, the models studied to elucidate the basic underlying properties can be classified in two groups. The first one considers struts as simple strings [21], while in the second the bending stiffness of the struts is also taken into account $[2,8,10,11,22-27]$. In both groups it can be distinguished between affine and nonaffine regimes depending on whether the deformation is ideally homogeneons or not, respectively $[3,4,8]$. The concept of affinity is very important since it gives an idea of how far the deformation will be homogeneonsly distribnted. Conseqnently, once a network is proved to be affine, it is easier to describe it with a simple model. Apart from this, in the second group two regines are identified: the stretchingcompression dominated one and the bending-doninated one $[3,4,8,24]$. The regine ranges and the transitions between thein have been studied in terms of parameters such as density, connectivity, relative bending stiffness, etc.; however, 
how the energy is distributed in the struts when a deformation is applied to the network has not been analyzed yet.

In this paper the correlation between the elastic energy in the struts and their orientation with respect to the loading direction is investigated. This study is carried out as a function of (a) the struts connectivity and (b) the relation between the stretching and bending stiffness. The interconnection between this study and the former properties in terms of affinity and stretching-bending deformation are determined in detail. It will be seen that all the three aspects, although being related to each other, have no functional dependence between them. In addition to the general findings, the approach using the energy distribution helps us to better understand the reorganization of biological materials. So, in the case of the cytoskeleton the filament orientation correlates with their deformation energy, and this, in turn, is known to be related to the stability of the filaments [28-30], so that the bundle formation process can be envisaged. In the case of the trabecular bone, this energy approach gives a simpler mechanical viewpoint of Wolf's law.

\section{MODEL DESCRIPTION}

The structures analyzed in this work are two-dimensional random networks of struts built in a square. The connectivity $C$, defined as the number of struts that converge in one node, is kept constant. Under this assumption, $C$ can only take the following values: 3,4 , and 6 . In the case of a regular network, these values refer to struts forming hexagons (like in a honeycomb), squares, and triangles, respectively. The disordered networks were generated by a random displacement of the nodes of such regular lattices. The nodes were consecutively moved to achieve randomness with the only restriction that the struts were not allowed to intersect. This process of moving the nodes was repeated ten times over the whole network, yielding to the random network in its unstretched configuration. Figure 1(a) shows one example with $C=4$.

In most of the previous works the two-dimensional networks were created by randomly introducing straight or curved segments of fixed length $[3,5,10,21,24,26,31,32]$. In those networks the connectivity is 4 in the majority of the nodes since the nodes stem from the intersection of two segments and the connectivity is 2 or 3 in some nodes if the not-linked extremes of the segments are removed. On the contrary, the networks obtained in this work allow investigating separately the effect of the connectivity and the effect of the relative thickness of the struts, which is directly related to the density of the network for a given connectivity. Besides, in those networks the direction of adjacent struts is correlated from the beginning since these stem from fragmented segments, while in the present case the direction of all struts is completely independent. From this point of view, these networks may represent a closer two-dimensional approach to biological materials.

For each lattice type (connectivity $C=3,4$, and 6) four different networks were built with a total number of struts $N$ approximately equal to $90,210,440$, and 580 . For all lattices the mean length of the struts $L_{m}$ was computed. In the network, each node has three degrees of freedom: the displace- (a)

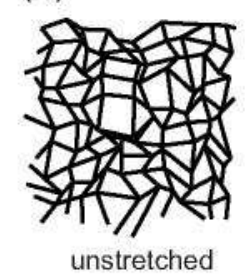

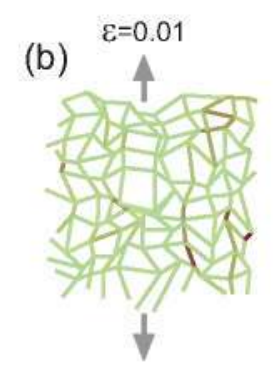

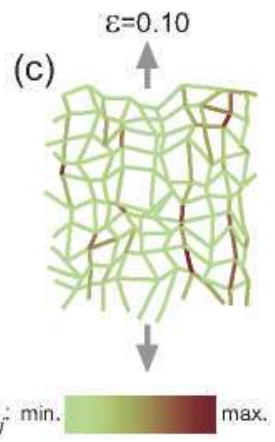

(d)

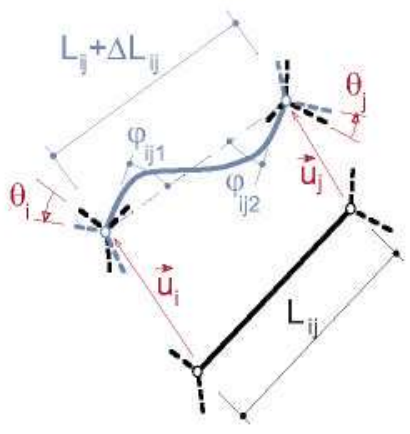

FIG. 1. (Color online) Example of a network of connectivity $C=4$, with $N=200$ struts and $r / L_{m}=0.02$ : (a) unloaded, (b) under a stretching strain $\varepsilon=0.01$, and (c) under $\varepsilon=0.10$. The calibration bar indicates the elastic energy of the struts per unit volume $w_{i j}$. (d) Sketch of a deformed strut between two generic nodes $i$ and $j$, indicating the displacement and the rotation angle of the nodes.

ments $u_{i x}, u_{i y}$ and the rotation angle $\theta_{i}$. Figure 1 (d) shows schematically the displacement and rotation of two neighbor nodes. The struts are considered to be linear elastic, bending as Euler-Bernoulli beams [33]. The cross section of all struts is equal for each simulated case. The geometric properties of the cross section are the area, $A$, and the moment of inertia, $I$. The latter is equal to $A r^{2}$, where $r$ is the radius of gyration. Young's modulus of the material is $E_{s}$. The elastic energy, $W_{i j}$, stored in a strut between nodes $i$ and $j$ is given by

$$
W_{i j}=\frac{1}{2} \frac{E_{s} A}{L_{i j}} \Delta L_{i j}^{2}+\frac{1}{2} \frac{4 E_{s} I}{L_{i j}}\left(\varphi_{i j 1}^{2}+\varphi_{i j 1} \varphi_{i j 2}+\varphi_{i j 2}^{2}\right),
$$

where $\Delta L_{i j}$ is the change in length of the strut and $\varphi_{i j 1}$ and $\varphi_{i j 2}$ are the rotation angles of nodes $i$ and $j$ with respect to the segment $i j$ in the deformed state [see Fig. 1(d)]. This model corresponds to elastic beams of isotropic material, where only bending and stretching or compression are considered. The buckling of the struts is not considered. The longitudinal stiffness of the struts $K_{l}=E_{s} A / L_{i j}$ and the bending stiffness $K_{b}=4 E_{s} I / L_{i j}$ are related by the expression $K_{b} / K_{l}=4 r^{2}$. The second term in Eq. (1) has been deduced from the stiffness matrix of the beam taking into account that the bending energy is fully characterized by the mentioned rotation angles. Equation (1) can be written as

$$
W_{i j}=\frac{1}{2} K_{l}\left[\Delta L_{i j}^{2}+4 r^{2}\left(\varphi_{i j 1}^{2}+\varphi_{i j 1} \varphi_{i j 2}+\varphi_{i j 2}^{2}\right)\right] .
$$

This linear model is of general validity, and the ratio $4 r^{2}$ between bending and longitudinal stiffness can be deduced 
by other considerations different from elasticity of homogeneous beams of isotropic material.

The final equilibrium state of a network that has been stretched is calculated by an iterative process that minimizes the total energy $W_{t o t}=\Sigma_{i j} W_{i j}$ by balancing the forces at the nodes. The energy of a single strut, $W_{i j}$, is computed from these parameters, as well as the energy per unit volume $w_{i j}=W_{i j} /\left(A L_{i j}\right)$. Further, the stretching-compression energy and the bending energy are evaluated for each strut and for the whole network.

In this work, we aimed at obtaining fundamental insights into the basic properties of disordered elastic networks; the loading condition studied is uniaxial, stretching along the $y$ axis. Periodic boundary conditions are applied to the network. Two cases of stretching strain $\varepsilon$ are considered: 0.01 and 0.10 (i.e., $1 \%$ and $10 \%$, respectively). Figures 1 (b) and 1(c) show an example of a deformed network under both strain levels. The elastic energy of the struts per unit volume $w_{i j}$ is indicated in a color scale.

The properties of the network depend on the architecture of the lattice — characterized by the connectivity $C$ - the elastic properties of the material, and the geometrical properties of the cross section of the struts. As a result, the elastic behavior of the struts is determined by $K_{l}$ and $r$. Only dimensionless parameters are used in the analysis, and the results are displayed as a function of the connectivity $C$ and the dimensionless parameter $r / L_{m}$. This last parameter quantifies the relation between bending stiffness and stretching stiffness.

\section{RESULTS AND DISCUSSION}

\section{A. Energy distribution}

The elastic energy in the struts is analyzed first for a stretching strain $\varepsilon=0.10(10 \%)$. Figure 2 shows the dimensionless elastic energy $w_{i j} / w_{m}$ (elastic energy per unit volume divided by the mean value) versus the orientation of the struts. The struts' energy was computed individually for every orientation, resulting always in similar distributions where the number of struts with energy around a certain value diminishes approximately linearly with the energy. The results for $r / L_{m}=0.170$, i.e., very thick struts [Figs. 2(a)-2(c)], and for $r / L_{m}=0.006$, i.e., thin struts [Figs. 2(d)-2(f)], represent statistical values computed from the whole ensemble of struts of the four networks. The average values are plotted together with the standard deviations. Both parameters display comparative values, which is a consequence of the approximately linear decay in the number of struts with the energy. In the first case, with $r / L_{m}$ $=0.170$, an upward trend between the orientation and the elastic energy in the struts is observed for the three values of connectivity, indicating that the higher the average value of $w_{i j}$ for a given strut is, the more aligned it is in the stretching direction. However, for the second case, with $r / L_{m}=0.006$, the increasing trend is less pronounced for $C=4$ and $C=6$, and it becomes even decreasing for $C=3$, so that the struts perpendicular to the stretching direction have a higher energy. These graphs evidence the existence, at least under cer-

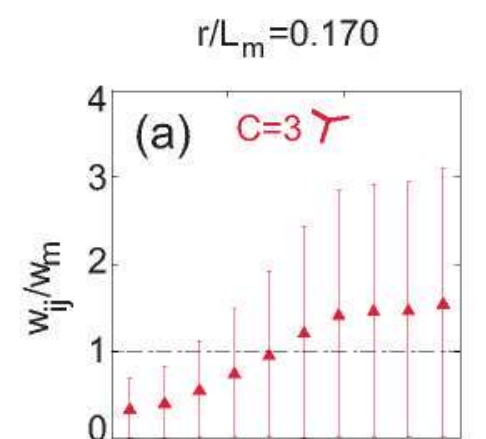

$r / L_{m}=0.006$
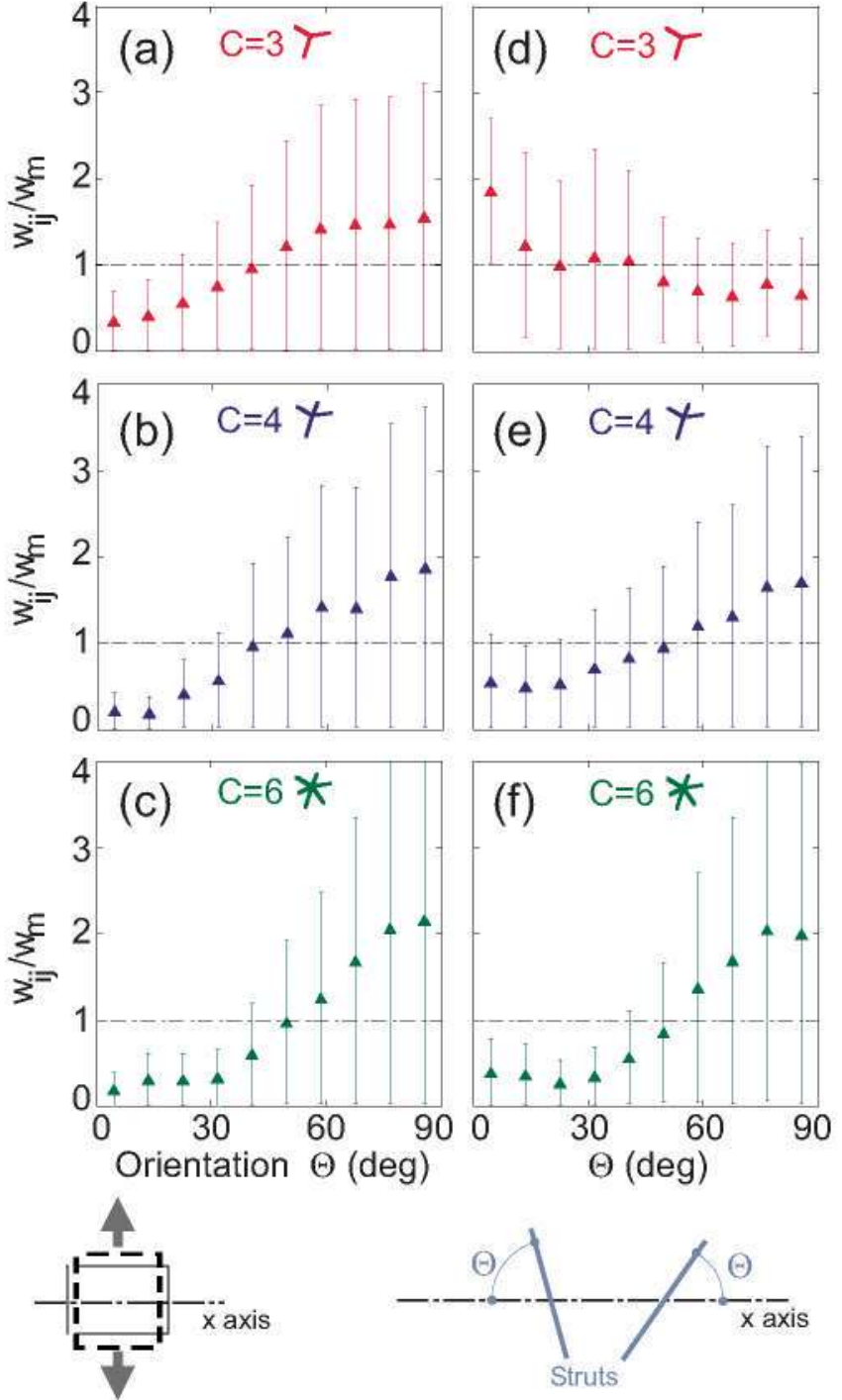

FIG. 2. (Color online) Average elastic energy versus orientation of the struts at stretching strain $\varepsilon=0.10$ for the three connectivities $(C=3,4$, and 6$)$ and two values of relative bending stiffness $\left(r / L_{m}=0.170\right.$ and 0.006$)$. The elastic energy is expressed as elastic energy of the struts per unit volume $w_{i j}$ divided by the mean value $w_{m}$. The struts are divided in ten groups between orientation $0^{\circ}$ (struts parallel to the $x$ axis) and $90^{\circ}$ (struts perpendicular to the $x$ axis, i.e., aligned to the loading direction). Error bars indicate standard deviation.

tain conditions, of a correlation between the expected energy and the orientation of the struts.

In order to analyze the range of conditions for which this dependency exists, the linear regression between $w_{i j} / w_{m}$ and the orientation was calculated. The slope $S$ obtained is displayed in Fig. 3 as a function of $r / L_{m}$ for the three values of connectivity and for the two stretching levels considered. At higher stretching levels $S$ becomes larger, indicating an increase in the energy difference between the struts aligned parallel and perpendicular to the stretching direction. This preferential stimulation of the struts is relevant for the directional remodeling processes in biomaterials, as it will be dis- 


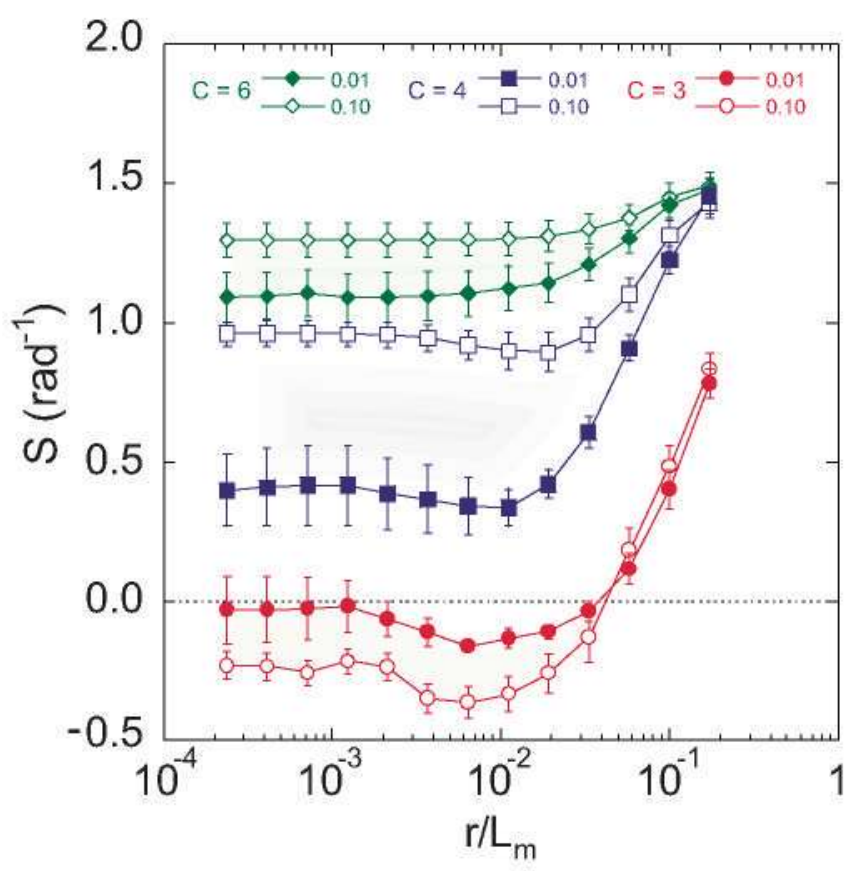

FIG. 3. (Color online) General dependence between the dimensionless elastic energy per unit volume $w_{i j} / w_{m}$ and the orientation of the struts for the three cases of connectivity and the two cases of stretching strain. This is estimated by the slope $S$ of the linear regressions considering the orientation angle in radians. $S \neq 0$ means that the expected elastic energy for a strut depends on its orientation. Mean value and standard deviation computed for the four networks calculated at each condition.

cussed in Sec. III B. On the other side, in the particular case of low connectivity and very low bending stiffness $(C=3$ and $\left.r / L_{m}<0.04\right) S$ becomes even negative so that the average elastic energy $w_{i j}$ reaches the highest values on transversal struts. Further, it is noteworthy that $S$ decreases as $r / L_{m}$ diminishes and it even vanishes to zero in the case of $\varepsilon=0.01$, indicating that the elastic energy is distributed over all struts independently of their orientation. On the other hand, as the struts become stiffer in bending, i.e., larger values of $r / L_{m}$, the slope $S$ reaches the highest values for the three connectivities, and thus the struts oriented in the direction of stretching have a higher probability to present the highest values of elastic energy per unit volume. It is noteworthy that for the largest values of $r / L_{m}$, i.e., high relative bending stiffness, $S$ converges to a saturation value independently of the stretching level. In connection with this result, in Sec. III C it will be shown that the fraction of stretching energy has a similar value in this region for all the cases.

\section{B. Biological materials}

This result is useful to interpret the particular phenomenon of remodeling observed in the trabecular bone (Wolf's law). Even though the thickness of the trabeculae varies in a certain range, the average ratio $r / L_{m}$ can be estimated in the order of 0.1 from images obtained by microcomputed tomography $[34,35]$. A bone undergoes loading and unloading due to the daily activity and, considering the simplest case of uniaxial loading, the maximum elastic energy in each strut correlates with its orientation as shown previously. According to Fig. 3, the trabeculae oriented close to the loading direction store, on average, a larger elastic energy per unit volume than those oriented transversally. This difference in mechanical strain, depending on the orientation of the trabeculae, leads to a differentiated response of the involved osteocytes (the mechanosensing cells in bone [20]). Bones undergo a continuous growth-resorption process, which is modulated by osteocytes. The ones that are subjected to a large fluctuation of strain are significantly stimulated and consequently promote the reinforcement of the struts nearly aligned with the loading direction, while, on the contrary, the low stimulus of the osteocytes in the transversal trabeculae favors their thinning. The presented model provides a simpler approach to understand the remodeling of bone based exclusively on the trabeculae orientation.

The results in Fig. 3 are also applicable to the biological model of the cytoskeleton. Its microstructure is subjected to remodeling driven by mechanical loading and by internal biochemical active processes [8]. Two systems that have attracted special attention are the spectrin cortical cytoskeleton of red blood cells [36,37] and the actin network in endothelial cells $[28,29,38]$. It has been observed that the actin filaments align perpendicularly to a stretching deformation applied cyclically when it is large enough. Consequently, the actin filaments are organized in bundles called stress fibers $[28,29,38]$. In order to analyze the evolution observed from the initially disordered actin network to the final fibers with the presented network model, the ratio $r / L_{m}$ needs to be determined. The ratio $r / L_{m}$ is estimated to be $\sim 0.01$ for F-actin networks from the following experimental values: $E I=7 \times 10^{-26} \mathrm{~N} / \mathrm{m}^{2}$ [39], $E A / L=35 \times 10^{-3} \mathrm{~N} / \mathrm{m}^{-1}$ [40], and $L_{m}=2.5 \times 10^{-7} \mathrm{~m}[41]$. According to Fig. 3, a plausible interpretation of the bundle formation can be as follows: assuming that the actin filaments cannot support deformations over a certain threshold of energy [28-30] and that they have a connectivity large enough to guarantee $S>0$ (i.e., larger than 3 if the system can be described by a two-dimensional network), the actin filaments transverse to the stretching will remain after the disassembly process since these are the ones that store the lowest elastic energy per unit volume. Besides, a stretching level sufficiently high is needed to achieve remodeling of actin bundles $[28,29,38]$. This suggests that the remodeling is promoted first at conditions with sufficiently high $S$ values, i.e., where the struts oriented in the stretching direction are more effectively stimulated. The model can also be applied to the case of the cortical cytoskeleton of red blood cells. This material has been modeled as a twodimensional network of connectivity $C=6$, defining a threshold of deformation energy above which the struts break [36]. As the struts with the higher deformation energy are aligned in the stretching direction, these are the ones that break preferentially. Thus, the remodeling process induced by stretching would lead to a network in which perpendicular struts are predominant.

\section{Bending-stretching transition and affinity}

In the analysis above the elastic energy is considered without distinguishing the two components involved in the 
(a)

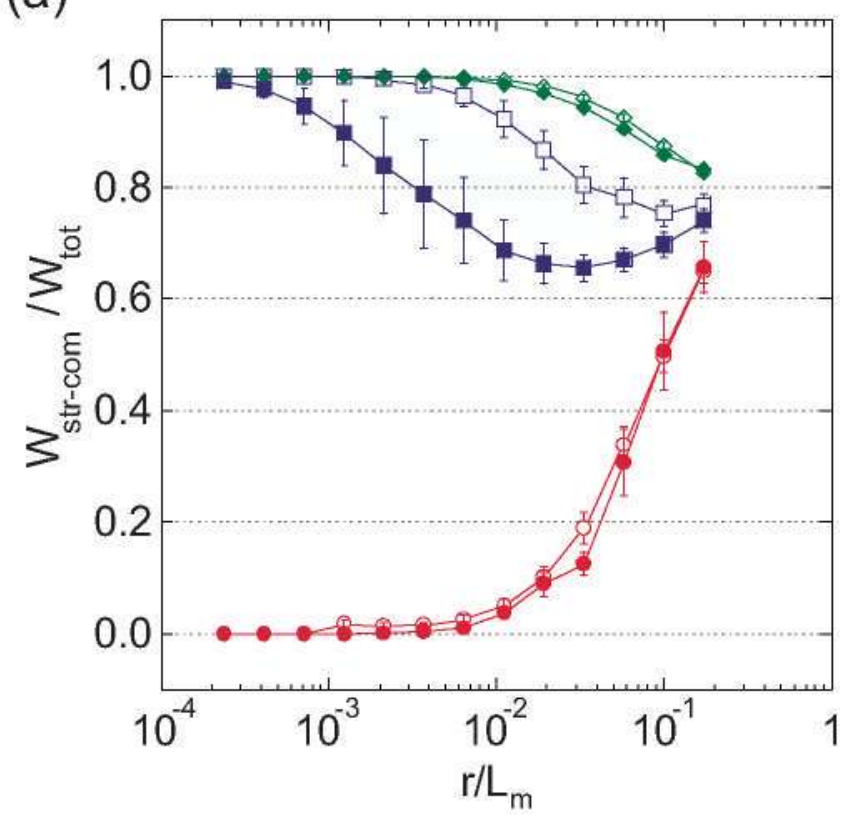

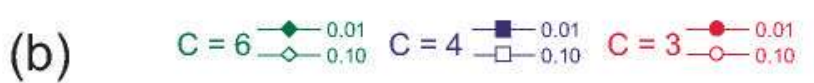

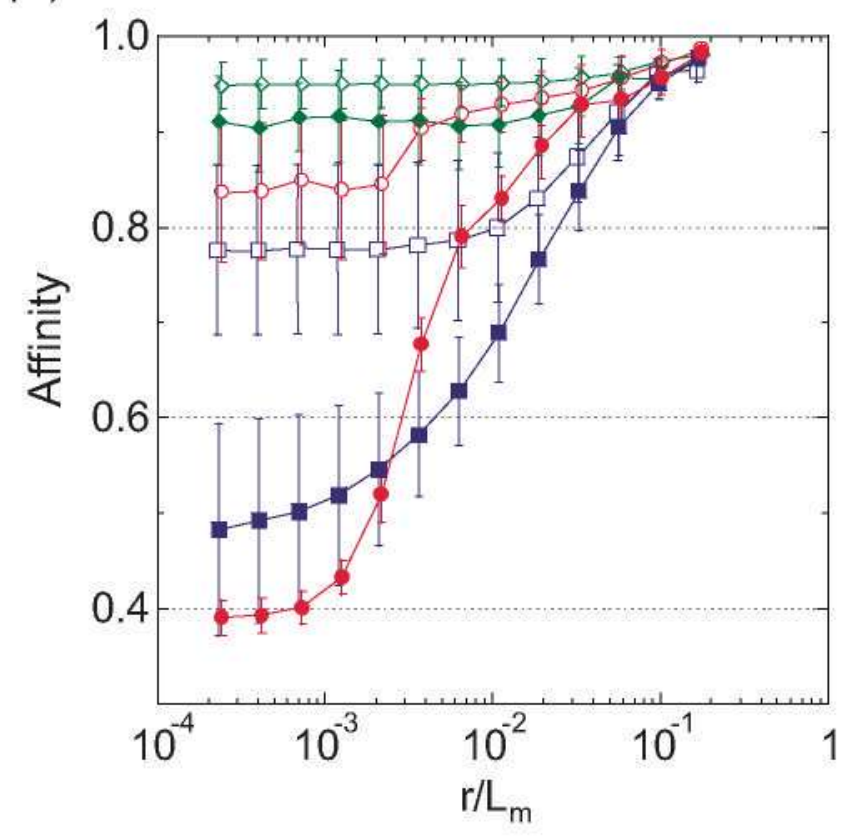

FIG. 4. (Color online) (a) Ratio of stretching or compression energy, $W_{s t r-c o m} / W_{t o t}$, as a function of the relative bending stiffness, $r / L_{m}$. (b) Standard correlation coefficient between the computed relative displacement and the calculated affine relative displacement $\Delta r_{k l}$ of every two points as a function of $r / L_{m}$. It becomes equal to 1 when the deformation is affine. Mean value and standard deviation computed for the four networks calculated at each condition.

total energy of the network: (a) the stretching-compression energy, $W_{s t r-c o m}$, and (b) the bending energy, $W_{\text {bend }}$. The relative contribution of these two energies is displayed in Fig. 4(a) as a function of $r / L_{m}$. When $W_{\text {str-com }}$ dominates, the ratio $W_{\text {str-com }} / W_{\text {tot }}$ is $>0.5$; on the contrary, it is $<0.5$ when $W_{\text {bend }}$ is larger. It is seen that for the connectivities $C=4$ and
6 , the energy is stored mainly in the form of stretchingcompression. In contrast, the bending component is the dominant one for the connectivity $C=3$, except for the cases when the relative bending stiffness is very high $\left(r / L_{m}\right.$ $>0.1$ ). The case $C=4$ represents an intermediate state between $C=3$ and $C=6$, and only in this particular case the fraction of stretching-compression energy depends significantly on the degree of deformation. Additionally, it is observed that for low values of $r / L_{m}$ the energy is stored basically in only one of the types. As $r / L_{m}$ increases, the counterpart component raises continuously, so that for the largest values of $r / L_{m}$ all the three connectivities tend to similar ratios, namely, between $65 \%$ and $85 \%$.

Besides the orientation dependence of the struts and the relative components of the total energy, the affine or nonaffine deformation nature of the networks is analyzed. The assumption that the deformation field is affine has allowed building simple quantitative theories for elastomers, while its applicability in the case of semiflexible polymers is under debate $[3-5,8]$. The affine or nonaffine deformation is analyzed by the standard correlation coefficient [8], which relates the computed and the corresponding affine relative displacement $\Delta r_{k l}$ for all the pairs of nodes $k, l$. Figure $4(\mathrm{~b})$ displays the affinity coefficient (equal to 1 for complete affinity) as a function of $r / L_{m}$. For very high values the affinity is very close to 1 , independently of connectivity and stretching. In the case of connectivity $C=6$, the affinity is very high in any case. On the contrary, the affinity drops rapidly with decreasing values of $r / L_{m}$ in the cases of lower connectivity and the smaller deformations from the initial status. Therefore, in agreement with previous findings [5], the networks display more affine behavior for higher deformations. This result indicates that small deformations lead to a relative homogeneous rearrangement of the network, while large deformations yield necessarily to a predominant directional stretch of the network. Further, it should be noted that Fig. 4 points out that the affinity is not necessarily linked to the stretching or compression regime reported by other authors $[5,8]$.

In previous works, both the stretching or bending contribution and the affinity of the deformation were studied as a function of the mean connectivity [8] or alternatively as a function of dimensionless parameters, which depend simultaneously on the relative bending stiffness and the mean connectivity [3-5]. In these studies a nonaffine to affine transition was found between the bending-dominated low connectivity regime and the stretching-dominated high connectivity one, in agreement with the results in Fig. 4.

The three aspects considered to study the deformation of disordered networks are summarized in Table I: the orientation dependence, the fraction of stretching energy, and the affinity. In the case of connectivity $C=6$, all three parameters have high values independently of the relative bending or stretching stiffness. In contrast, for connectivity $C=3$ high values are reached only for large $r / L_{m}$, and these decrease gradually for smaller values of $r / L_{m}$. The situation for connectivity $C=4$ is intermediate; the stretching energy fraction is close to the case of $C=6$; instead, the affinity is similar to the case $C=3$, and finally the orientation dependence lies between the other two cases. Therefore, although there is no functional relation between the three aspects, it is observed 
TABLE I. Summary of the network behavior depending on the three considered parameters: the orientation dependence, the fraction of stretching energy, and the affinity. The symbols indicate the values of the parameters: (++) high, (+) medinm. (0) low. and (-) negative.

\begin{tabular}{|c|c|c|c|c|c|c|}
\hline \multirow{2}{*}{$\begin{array}{l}\text { Connectivity } \\
\text { (C) }\end{array}$} & \multicolumn{2}{|c|}{$\begin{array}{l}\text { Orientation dependence } \\
\qquad(S)\end{array}$} & \multicolumn{2}{|c|}{$\begin{array}{l}\text { Fraction of strelching energy } \\
\qquad\left(W_{\text {str-cont }} / W_{t o t}\right)\end{array}$} & \multicolumn{2}{|c|}{ Affinity } \\
\hline & Low $r / L_{m}$ & Higln $r / L_{m}$ & Low $r / L_{n t}$ & High $r / L_{n t}$ & Low $r / L_{m}$ & High $r / L_{m}$ \\
\hline 3 & - & + & 0 & + & $0+$ & ++ \\
\hline 4 & + & ++ & ++ & + & $0+$ & ++ \\
\hline 6 & ++ & ++ & ++ & + & ++ & ++ \\
\hline
\end{tabular}

that high connectivity always relates wilh a high grade of aftinity and a dominant stretching energy. On the contrary, for low connectivity these values decrease gradually with the relative bending or stretching stiffness.

\section{SUMMARY AND CONCLUSIONS}

The deformation of two-dimensional randomly disordered networks during the application of a nniaxial stretching is studied taking into consideration a fixed connectivity. In a two-dinensional network there are only three possible connectivities: $C=3,4$, and 6 . The energy of the struts is evaluated in these kinds of networks as a function of the struts' orientation for a fixed relative bending stiffness $r / L_{n}$. Two stretching levels are studied. The trends in (a) orientational energy distribution in the struts, (b) fraction of stretchingbending energy, and (c) affinity of deformation are investigated as a function of the relative bending stiffness and the connectivity. For low and medinm $r / L_{n}$ valnes-first regime-the directional dependence of the energy remains approximately constant for each connectivity and stretching level. Struts aligned in the stretching direction present a larger energy for $C=4$ and 6 . In contrast, $C=3$ shows a lower orientational dependence of the struts' energy. It is remarkable that in this case of $C=3$ the orientational dependence is even negative, so that the perpendicular struts store the higher values of energy. For large $r / L_{n}$ valnes-second regime-an increasing relative bending stiffness leads, for all three cases of connectivity, to a preferential accnmulation of energy in the strnts oriented in the stretching direction. The deformation mode of the struts can also be classified into the mentioned two regimes. In the first regine and for $C=3$ the energy is dominantly stored as bending energy, opposite to $C=6$ where it is basically of stretching or compression nature. $C=4$ represents an intermediate state between $C=3$ and 6 . In the second regime the strain is a mixture of stretching or compression and bending; here, all three connectivities tend to converge toward a 0.7 ratio. Finally, it has been shown that the affinity is very high for $C=6$, while for $C=4$ and 3 the degree of affinity increases with the stretching level. With the increase of relative bending stiffness the affinity raises almost to unity for all three connectivities, indicating an almost homogeneons strain among the whole network.

Although for the sake of clarity the studied model is twodimensional and only uniaxial stretching is analyzed, the trends found can be reasonably extrapolated to the understanding of three-dimensional networks. This is of particular interest for biological materials, such as, for exanple, the trabecnlar bone and the cytoskeleton. Both are constituted by a disordered network, which nndergoes a remodeling process in order to adapt the microstructure to the external mechanical loading conditions. In the case of the trabecnlar bone, the estimated relative bending stiffness is so high that, according to the presented model, in average the struts oriented in the direction of the loading store the largest energy. Consequently, the related bone cells (osteocytes) have a larger mechanical stimulus and subsequently these struts are reinforced. The resulting network follows then the Wolf's law. On the other hand, the cytoskeleton represents the same model with opposite resnlt. In this case, when the network of actin filaments is subjected to large stretching and assuming that the filaments with larger energies are disassembled, one obtains at the end the experimentally observed bnndles of filaments transverse to the stretching direction. Consequently, the two-dimensional model described above is able to provide a simple explanation of the remodeling of biological materials based uniquely on mechanical aspects.

\section{ACKNOWLEDGMENT}

The author thanks Encana G. Víllora for her time invested in insightful discussions and reviewing the text.
[1] K. E. Kasza. A. C. Rowat, J. Y. Lin, T. E. Angelini, C. P. Brangwynne, G. H. Koenderink, and D. A. Weitz, Curr. Opin. Cell Biol. 19, 101 (2007).

[2] F. C. MacKintosh, J. Kas, and P. A. Janmey, Phys. Rev. Lett.
75,4425 (1995).

[3] D. A. Head, A. J. Levine, and F. C. MacKintosh, Phys. Rev. Lett. 91, 108102 (2003).

[4] D. A. Head, A. J. Levine, and F. C. Mackintosh, Phys. Rev. E 
68. 061907 (2003).

[5] P. R. Onck, T. Koeman, T. van Dillen, and E. van der Giessen, Phys. Rev. Lett. 95, 178102 (2005).

[6] C. Storm, J. J. Pastore. F. C. MacKintosh, T. C. Lnbensky, and P. A. Janmey, Natnre (London) 435, 191 (2005).

[7] F. Pampaloni, G. Lattanzi, A. Jonas. T. Snrrey. E. Frey. and E. L. Florin, Proc. Nall. Acad. Sci. U.S.A. 103, 10248 (2006).

[8] G. A. Buxton and N. Clarke, Phys. Rev. Lett. 98, 238103 (2007).

[9] O. Chaudhun, S. H. Parekh, and D. A. Fletcher, Nature (London) 445,295 (2007).

[10] S. Roy and H. J. Qi. Phys. Rev. E 77, 061916 (2008).

[11] G. A. Bnxton, N. Clarke, and P. J. Hnssey, Express Polym. Lett. 3, 579 (2009).

[12] G. Bao and S. Suresh, Nature Mater. 2, 715 (2003).

[13] W. W. Almed, T. Wolfram, A. M. Goldyn, K. Bruellhoff, B. A. Rioja, M. Moller, J. P. Spatz, T. A. Saif, J. Groll, and R. Kemkemer, Biomaterials 31, 250 (2010).

[14] A. Zemel, F. Rehfeldt, A. E. X. Brown. D. E. Discher, and S. A. Satran. J. Phys.: Condens. Matter 22. 194110 (2010).

[15] D. Miznno. C. Tardin. C. F. Schınidt. and F. C. MacKintosh. Science 315, 370 (2007).

[16] S. Jungbater, H. Gao, J. P. Spatz, and R. Kenkemer, Biophys. J. $95,3470(2008)$

[17] J. Wolff, Das Gesetz der Transfomation der Knochen (A. Hirchwild, Berlin, 1892) [translated as The Low of Bone Re. modeling, edited by P. Maquet and R. Furlong (Springer, Berlin, 1986)].

[18] P. Fratzl and R. Weinkanner. Prog. Mater. Sci. 52, 1263 (2007).

[19] H. M. Frost. Anat. Rec. 219, 1 (1987).

[20] R. Hniskes. R. Rnimerman. G. H. van Lenthe. and J. D. Janssen, Natnre (London) 405. 704 (2000).

[21] M. Latva-Kokko, J. Makinen, and J. Timonen, Phys. Rev. E 63. 046113 (2001).

[22] M. J. Silva and L. J. Gibson, Bone (N.Y.) 21, 191 (1997).

[23] S. Vajihala, A. M. Kraynik, and L. J. Gibson, J. Biomech. Eng.
122. $511(2000)$.

[24] J. Wilhelm and E. Frey, Phys. Rev. Lett. 91, 108103 (2003).

[25] D. A. Head, A. J. Levine, and F. C. MacKintosh, in Slow Dynamics in Complex Systems, edited by Michio Tokuyama and Irwin Oppenheim, AIP Conf. Proc. No. 708 (AIP, Melville. NY. 2004).

[26] C. Henssinger and E. Frey, Phys. Rev. Lett. 96, 017802 (2006).

[27] E. M. Hnisman. T. van Dillen. P. R. Onck, and E. Van der Giessen, Phys. Rev. Lett. 99, 208103 (2007).

[28] R. Kaunas and H. J. Hsu, J. Theor. Biol. 257, 320 (2009).

[29] J. H. C. Wang, J. Theor. Biol. 202, 33 (2000).

[30] R. Krishnan et al., PLoS ONE 4, e5486 (2009),

[31] M. Kellomäki, J. Astrom, and J. Timonen, Phys. Rev. Lett. 77. 2730 (1996).

[32] M. Latva-Kokko and J. Timonen. Phys. Rev. E 64. 066117 (2001).

[33] A. Ghali. A. M. Neville. and T. G. Brown. Structural Analysis. A Uniffied Classical and Matrix Approach (Spon Press, London, 2003).

[34] T. Uchiyama. T. Tanizawa. H. Mnranatsn, N. Endo. H. E. Takahashi, and T. Hara, Bone (N.Y.) 25. 487 (1999).

[35] A. Nazarian, M. Stauber, D. Zurakowski, B. D. Snyder, and R. Muller, Bone (N.Y.) 39. 1196 (2006).

[36] J. Li, G. Lykotrafitis, M. Dao, and S. Suresh, Proc. Natl. Acad. Sci. U.S.A. 104, 4937 (2007).

[37] N. S. Gov, Phys. Rev. E 75. 011921 (2007).

[38] P. C. Dartsch and H. Hammerle, Eur. J. Cell Biol. 41, 339 (1986)

[39] F. Gittes and F. C. MacKintosh. Phys. Rev. E 58. R1241 (1998).

[40] X. M. Liu and G. H. Pollack, Biophys. J. 83, 2705 (2002).

[41] M. L. Gardel, F. Nakamura, J. H. Hartwig, J. C. Crocker, T. P. Stossel, and D. A. Weitz, Proc. Nall. Acad. Sci. U.S.A. 103. $1762(2006)$. 